\title{
Selection of Appropriate Software Architecture Style with Square Spline in Style based Systems
}

\author{
Hamidreza Hasannejad, Homayun Motameni and Hossein Nematzadeh ${ }^{*}$ \\ Department of Computer Engineering, Islamic Azad University, Sari Branch, Iran; \\ nematzadeh@iausari.ac.ir
}

\begin{abstract}
Software complexity and size growth are two factors that yield to have architecture as a significant designing tool. Architecture explains a system's responsibility for stockholders' functional and non-functional requirements. In style based software systems, achieving appropriate software architecture style is dramatically important. Thus, this research tries to analyze each architectures's style behavior and also stockholders' desired model (in the form of a mathematical function diagram) implementable using Square Spline Interpolation. An algorithm was presented and then Square Spline Interpolation was applied. All the calculations and plotting were done using MATLAB. This makes the appropriate architecture style to be selected among all architecture style with the help of the Desired Model (DM) curve. The result of this research confirms the previous statistical and matrix based method however the presented methodology has less computation and more accuracy specially where the candidate styles are really close to each other. Having stockholders' all non-functional requirements responded is out of the question, because some of them are against each other. The proposed methodology can create a trade-off amongst non-functional requirements.
\end{abstract}

Keywords: Non-functional Requirements, Square Spline Interpolation, Software Architecture Style

\section{Introduction}

Selection of an appropriate architecture style at the time of designing a software system has an important role to achieve a success in style based systems. Since this could help to use appropriate analysis models for predicting a favorite architecture specifications. Using frameworks and different architectural middle ware could save time and cost both at running and establishing the intended architecture. Moreover, because of the frequent use of architectural styles by architects, unwanted changes will be prevented. This policy has positive effects in the evolution of the intended architecture. The paper aims to select a software architectural style among the candidate styles with the help of Square Spline Interpolation.
Literature reveals different researches on style analysis and software architectural model evaluation in order to select the best model among the existing. Bosch et al. presented a method called arch designer ${ }^{4}$. Arch designer not only focused on evaluating of non-functional parameters and priorities scheduling through assigning weights but also concentrated on selection of the most appropriate architecture among existing architectures with the help of numeric methods. HoseiniJabali et al. first calculated the density using vectors and then selected the architecture with the highest density ${ }^{7}$. HoseiniJabali et al. used AHP algorithm in his proposed method. Correlation coefficient is one of the other ways to evaluate styles or architectural model which was used in the literature $\mathrm{e}^{1,3,8-12}$.

*Author for correspondence 


\section{Software Architectural Styles}

To software systems there are different methods for breaking systems to parts and relations. Some of these methods and architectures have been frequently proposed by architects for specified systems. The functionality and applicability of the mentioned methods and architectures have been approved and hence a set of architectures or architecture samples has been established to help architects in presenting architecture. Each of the architectures of the established set is defined and considered as an architecture style. The subject of architecture style has been discussed in recent years in many scientific research papers. This is because styles represent the knowledge and wisdom of skillful architects. This could help novice architects to design their architectures. Although the basic motivation for selecting an architecture style in special situations is regarding the reasoning and qualitative characteristics. The term architecture style was first identified by Wolf and Perry in 1992 and Garlan and Shaw introduced and illustrated it afterwards ${ }^{6}$. There are different definitions for architectural style which only some of them is presented below.

Perry presented a definition of the style in 1992 as follow: An architectural style defines a family of software systems regarding structural organization. Moreover, styles explain components and their relations and also demonstrate their using limitations and their compositional rules ${ }^{10}$.

Shaw presented such a definition in 1996: An architectural style is a description of components and their arrangement which includes the description of pattern of data transmission and controlling among components. An style also presents an informal description of advantages and disadvantages in case of use $\mathrm{e}^{6}$.

Busechmann et al. defined the style in 1996: An architectural pattern presents fundamental appearance from structural organization for software systems.

Clements defined "An architectural style is dedication of components and communication among them with each other along with a set of rules and limitations about how to use them".

Albin in 2003 presented a definition of the style as follow: An architectural style is kind of hyper model to determine a set of components and communication among them in order to determine a system based on its style ${ }^{2}$.

Communication among components is based on limitations that characterize how it can combine the components and links. Different architectural styles illustrate the difference between various design classes. This task is done by presenting experimental evidence through using each class regarding qualitative reasoning and having the properties of that class. Style can be considered as a set of rules regarding architecture. Architecture style is a set of rules to design which shows the different types of components and communication that can be used to create a system. Components regarding links, types of characters are architectural styles giving each of them. The basis in classifying of styles is the recognition of the controlling procedure and data transmission between components.

\section{Methodology}

The main objective of this research is to find the appropriate architectural style (the desired model) using Square Spline Interpolation. The proposed methodology is a quantitative approach in which the diagram of each style would be produced and then matched with the desired model to select the most appropriate curve. In the proposed methodology, the architectural styles are compared based on their impact on non-functional parameters. Of course, the non-functional parameters' measurement is done quantitatively. An algorithm will be presented which helps to select the appropriate architectural style. MATLAB was used for calculations and plotting the curves. The proposed methodology has four stages. At first, the DM of stockholders should be determined. Next, the style's qualitative parameters have to be calculated quantitatively. Then, the curve diagram of square Spline will be conducted on all styles as well as the desired model of stockholders. Finally, after investigating the results and diagrams of software, the most efficient and appropriate architectural style that is the closest one to the DM of stockholders will be selected. The methodology is illustrated in Figure 1.

\subsection{Introduction and Calculation of Stockholders Requirements}

The first step is the calculation of the required qualitative parameters (non-functional parameters) with regard to stockholders and introducing them in the form of a vector. In order to find each of the indices of the vector we should consider all the requirements of all stockholders. Each of the non-functional parameters from the point of views of stockholders have different levels of importance e.g., the non-functional parameter of security could be very important from a point of view of a certain stockholder 


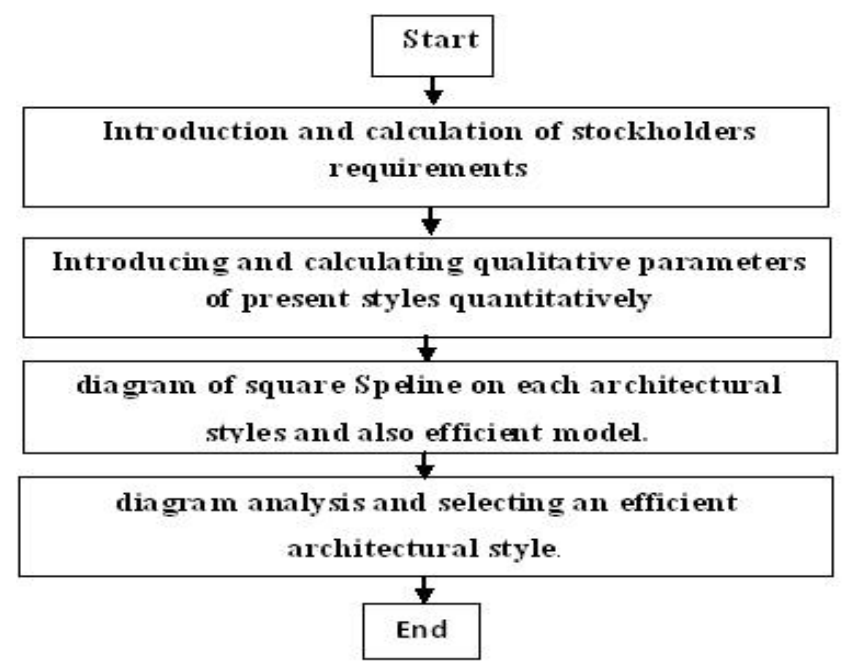

Figure 1. The stages of performing the selection of software architectural style.

and at the same time appeared unimportant or less important for another stockholder. Non-functional parameters can be converted to quantitative numbers using different methods. The methods that can be used in this paper are the interval scale methods. One simple and smart interval scale method is the one which was proposed by Babamir and Khabazian ${ }^{3}$ and defined as follows:

i) If a quality attribute has a negative effect for stockholder, we assign number 1 for it.

ii) If a quality attribute has positive effect for stockholder, we assign number 3 for it.

iii) And finally, if a quality attributes neither positive nor negative for stockholder (it is neutral for stockholder), we assign number 2 for it.

We use a very simple method to create vector of the requirements of stockholders. We take the average of each quality attribute. Table 1 showed a sample of a system composed of three stockholders with different requirements as well as the calculated vector of stockholder requirement.

Table 1. Calculation of DM

\begin{tabular}{lcccccc}
\hline \multicolumn{1}{c}{ Quality } & QA1 & QA2 & QA3 & QA4 & QA5 & QA6 \\
Stakeholders & & & & & & \\
\hline ST1 & 1 & 3 & 3 & 3 & 3 & 3 \\
ST2 & 2 & 3 & 3 & 3 & 1 & 3 \\
ST3 & 3 & 3 & 3 & 3 & 2 & 3 \\
DM & 2 & 3 & 3 & 3 & 2 & 3 \\
\hline
\end{tabular}

$\mathrm{QA}=$ Qualitative Attribute, $\mathrm{ST}=$ Stockholder, $\mathrm{DM}=$ Desired Model

\subsection{Introducing and Calculating Qualitative Parameters of Present Styles Quantitatively}

It is clear that the method of curve fitting vector is a mathematical way that makes it possible to obtain a continuous function get using quantitative separate points. To draw the diagram of such a continuous function using curve fitting vector at least two numeric dimensions (lengths( $\mathrm{x}$ ) and width(y)) are required. This is because to create the function mathematically in curve fitting vector method $\mathrm{X}$ and $\mathrm{Y}$ values are used directly. So, converting quality attributes (or qualitative parameters) to quantitative numbers is the first step before applying curve vector fitting.

One of the ways to represent the qualitative parameters is the tabular representation as was discussed in Table 1. Using the same method we try to assign numbers to architecture styles as follow:

i) If the architectural style has a negative effect upon a qualitative feature, we assign number 1 for it.

ii) If the architectural style has a positive effect upon a qualitative feature, we assign number 3 for it.

iii) And finally if the related architectural style has a neutral effect upon a qualitative feature, we assign number 2 for it.

The illustration of the proposed method is given in Table 2.

\subsection{Diagram of Square Spline on Each Architectural Styles and also DM}

At this stage, it was put an effort to determine a behavior for each of the architectural style. So each software architectural style and also the desired model have its own model. The values of $\mathrm{x}$ are qualitative parameters.

Table 2. Software architectural style parametric table

\begin{tabular}{lllllll}
\hline QA & QA1 & QA2 & QA3 & QA4 & QA5 & QA6 \\
Style & & & & & & \\
\hline A1 & 2 & 3 & 2 & 3 & 2 & 3 \\
A2 & 2 & 3 & 3 & 3 & 2 & 2 \\
A3 & 3 & 3 & 2 & 3 & 3 & 1 \\
\hline
\end{tabular}

$\mathrm{A}=$ Architecture style, $\mathrm{QA}=$ Qualitative Attribute 
Since in curve fitting methods the value of $\mathrm{x}$ is necessary to establish a continuous function, we assign a certain number to each parameter according to Table 3.

There are two types of values for each qualitative attribute in Table 3. We will investigate the difference between these numbers later in the paper. After converting qualitative parameters to quantitative values, we should apply the square Spline fitting upon each of the candidate styles and also DM in Table 2. To obtain more accurate result in problems, we usually use higher degree polynomials. However, using higher degree polynomials not only increases the number of calculated operation but also the results could also be unreliable because of truncating the results. Therefore, the piece wise method is used to achieve the most possible accurate result. With classifying the given interval $[a, b]$ to intervals of $[x i-1, x i]$ and roughly calculating the function using the lower degree polynomials, we can increase the accuracy and prevent the existence of unreliability in the results. The simplest approach here is the application of square Spline method. Equations 1 and 2 show the approach.

Figure 2 shows the two different ways for quantifying the qualitative parameters of Table 3.The diagram of different styles are different but the desired model of both are same which is A2.

Table 3. Validation of attributes

\begin{tabular}{lcccccc}
\hline $\begin{array}{l}\text { Qualitative } \\
\text { attributes }\end{array}$ & QA1 & QA2 & QA3 & QA4 & QA5 & QA6 \\
\hline $\begin{array}{l}\text { First validation } \\
\text { of X }\end{array}$ & 0 & 1 & 2 & 3 & 4 & 5 \\
$\begin{array}{l}\text { Second } \\
\text { validation of X }\end{array}$ & 5 & 3 & 1 & 4 & 2 & 0 \\
\hline
\end{tabular}

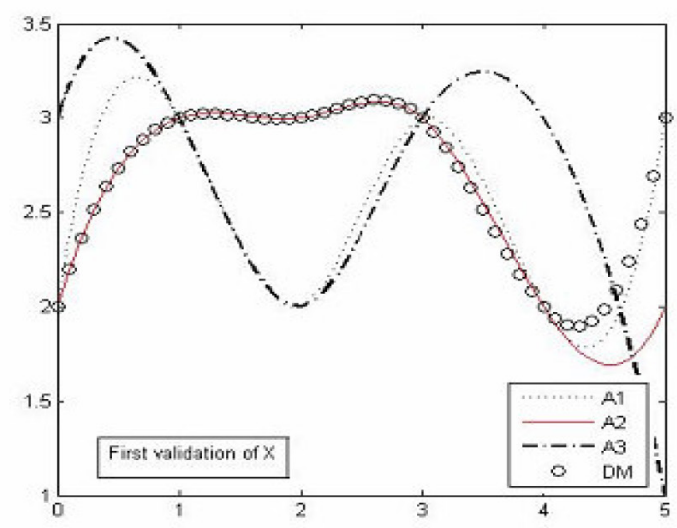

$$
\begin{aligned}
& A(x)= \frac{1}{6 h}\left(x_{i}-x\right)\left[\left(x_{i}-x\right)^{2}-h^{2}\right]+\frac{1}{6 h}\left(x-x_{i-1}\right) \\
& {\left[\left(x-x_{i-1}\right)^{2}-h^{2}\right] M_{i}+} \\
& \frac{1}{h}\left[\left(x_{i}-x\right) f_{i-1}+\left(x-x_{i-1}\right) f_{1}\right], \quad 1 \leq i \leq n \\
& M_{i-1}+4 M_{i}+M_{i+1}=\frac{6}{h^{2}}\left[f_{i+1}-2 f_{i}+f_{i-1}\right], \quad 1 \leq i \leq n-1
\end{aligned}
$$

a) $\mathrm{M}_{0}=\mathrm{M}_{\mathrm{n}}=0$

b) $M_{0}=M_{n}, M_{1}=M_{n+1}, f_{0}=f_{n}, f_{1}=f_{n+1}$

c) $2 \mathrm{M}_{0}+\mathrm{M}_{1}=\frac{6}{\mathrm{~h}}\left[\frac{\mathrm{f}_{1}-\mathrm{f}_{0}}{\mathrm{~h}}-\mathrm{f}_{0}\right]$,

$$
M_{n-1}+2 M_{n}=\frac{6}{h}\left[f_{n}-\frac{f_{n}-f_{n-1}}{h}\right]
$$

\subsection{Diagram Analysis and Selecting an Efficient Architectural Style}

Regarding Figure 2, the best style for the desired model of stockholders is A2. Because the A2 curve has the most compatibility with the desired model curve of stockholders. To prove this, we use the integral between curves. We calculate the area between the curve of each style and the curve of the desired model. The style which has the lowest difference in area with the desired model is the answer. The calculation process is as shown in Figure 3 and Equation 3.

$$
S_{j(x)}=\int_{a}^{b}\left|A_{j(x)}-D M(x)\right| d x \quad, \quad 1 \leq j \leq m
$$

In Equation 3, $\mathrm{Aj}(\mathrm{x})$ and $\mathrm{DM}(\mathrm{x})$ stands for each of the architectural style candidate function and the desired model function. The calculation and comparison results are shown in Table 4.

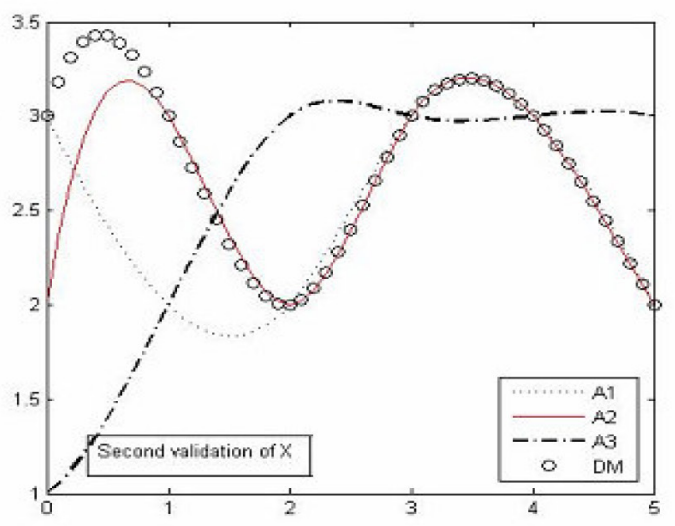

Figure 2. Diagram of square Spline result of each style and also DM of stockholders. 


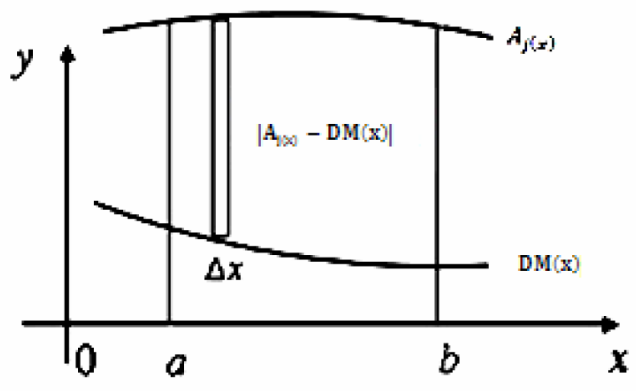

Figure 3. Area creating by two curve $\mathrm{Aj}(\mathrm{x}), \mathrm{DM}(\mathrm{X})$.

\begin{tabular}{|c|c|c|}
\hline $\begin{array}{l}\text { Best } \\
\text { chosen }\end{array}$ & Results & Values \\
\hline A2 & $\begin{array}{l}S=[16.62,4.09 \\
32.83] \\
S=[14.40,4.09 \\
50.45]\end{array}$ & $\begin{array}{l}{[0,1,2,3,4,5]} \\
{[5,3,1,4,2,0]}\end{array}$ \\
\hline
\end{tabular}

The input values in Table 4 are the inputs in Table 3. Based on these inputs the results were calculated with Square Spline Interpolation. According to the obtained results of architectural styles, A2 is the best selection for the efficient model mentioned which can be easily seen from Figure 2 and also calculated using Equation 3 and Figure 3. The proposed methodology benefits much when compare to previous methods as it is less computational complexity and more accurate in result especially where the candidate styles are really close to each other. In Figure 2 it is clear that A1 and A2 are close to each other but the proposed methodology finally selected A2 as the most appropriate style.

\section{Conclusion}

The qualitative parameters have basic role in software architecture type and can easily be converted in to quantitative values. Having these quantitative values and the proposed methodology it could be possible to select the appropriate architectural style which is close to the intended requirement of stockholders.

This paper used a new method in software architecture style under Spline curve diagram. The curve diagram methods were both mathematical and diagrammatic. Creating diagrams with those mentioned methods can help to investigate the area of each curve using integral. As a future direction of this research other forms of the curve including Least Squares Curve Fitting, Cubic Spline, Lagrange Interpolation, Newton Divided Difference, Hermite Interpolation, and Correlation Coefficient will be used for selecting models and software architecture style.

\section{References}

1. Astudillo H. Five ontological levels to describe and evaluate software architecture. Department de Informatics, Universidad Technical Federico SantaMaria Avda.España 1680, Valparaiso, Chile; 2004.

2. Albin ST. The art of software architecture: design methods and techniques. United States: John Wiley and Sons Ltd; 2003.

3. Babamir SM, Khabazian S. Evaluation of qualitative requirement analysis in software architecture. International conference of IT Knowledge; 2007; Mashhad, Iran.

4. Bosch J. Bengtsson PO, Smedinga R. Assessing optimal software architecture maintainability. Fifth European Conference on Software Maintainability and Reengineering; 2004.

5. Buschmann F, Meunier R, Rohnert H, Sommerlad P, Stal M. Pattern-Oriented Software Architecture: A system of Patterns. John Wiley \& Sons; 1996.

6. Garlan D, Shaw M. An introduction to software architecture". advances in software engineering and knowledge engineering, series on software engineering and knowledge engineering. World Scientific Publishing Company, Singapore, 1994.

7- HoseiniJabali F, Sharafi SM. Zamanifar K. A quantitative algorithm to select software architecture by tradeoff between quality attributes. World Conference on Information Technology; 2001. p. 1480-84.

8. Garlan D, Kim JS. Analyzing architectural styles with alloy. WorkshopontheRoleofSoftwareArchitectureforTestingand Analysis (ROSATEA); 2006.

9. Clements P, Bass FB, Garlan LD. Ivers J, Little R, Nord R, Stafford J. Documenting software architectures: views and beyond. Addison Wesley; 2007.

10. Perry DE, Wolf AL. Foundations for the study of softwarearchitectures. ACMSoftwareEngineeringNotes; 1999.p. 40-52.

11. Sharafi SM, Ghazvini GA, Emadi S. An analytical model for performance evaluation of software architectural styles. 2nd International Conference on Software Technology and Engineering (ICSTE).2010. p. 394-98.

12. Wang J, He X, Deng Y. Introducing software architecture specification and analysis in SAM through an example. Information and Software Technology; 1999. 